Digestive Surgery 中文目录2006年第23卷第5-6期

Table of Contents Vol.23, No. 5-6 2006

翻译 肖震宇 审校 陈孝平 (华中科技大学同济医学院附属同济医院肝脏外科中心)

Translated by Zhenyu Xiao and supervised by Xiaoping Chen, Hepatic Surgery Center of

Tongji Hospital Affiliated Tongji Medical College, Huazhong University of Science and

Technology

目前此翻译内容仅包括自2004年以来的《消化外科》杂志目录。

For the moment, this translation service includes only the Table of Contents from Digestive

Surgery and starts with the first issue of the year 2004.

社论 Editorial

281 剖腹手术中耻骨上置管引流的应用

Suprapubic Catheterization at Laparotomy

Johnson, C.D.

Dig Surg 2006;23:281-282

\title{
综述 Review
}

283 应用加强式定书钉式吻合器进行胃肠吻合：运用新技术以减少术后并发症

Buttressing of the Staple Line in Gastrointestinal Anastomoses: Overview of New Technology Designed to Reduce Perioperative Complications

Yo, L.S.F. ; Consten, E.C.J. ; Quarles van Ufford, H.M.E. ; Gooszen, H.G. ; Gagner, M.

Dig Surg 2006;23:283-291

经验交流 How I Do It

292 咽造口管: 一种简单的食管胃术后长期胃减压法

Tube Pharyngostomy: A Simple Method for Prolonged Intubation of the Foregut following Oesophagogastric Surgery

Katsoulis, I.E. ; Livingstone, J.I. ; Sagor, G.R.

Dig Surg 2006;23:292-295

296 胰十二指肠切除术后胰管对粘膜的三层胰空肠吻合术

Triple-Layer Duct-to-Mucosa Pancreaticojejunostomy after Pancreaticoduodenectomy Ibrahim, S. ; Tay, K.H. ; Launois, B. ; Tan, N.C.

Dig Surg 2006;23:296-302

\section{临床影像 Clinical Images}

303 结肠病变术前应用印度墨水定位后出现的并发症

Complication after Pre-Operative India Ink Tattooing in a Colonic Lesion

Singh, S. ; Arif, A. ; Fox, C. ; Basnyat, P.

Dig Surg 2006;23:303

论著 Original Paper 
304 胆囊切除术后良性胆道狭窄的长期观察结果

\section{Postcholecystectomy Benign Biliary Strictures - Long-Term Results}

Sikora, S.S. ; $\quad$ Pottakkat, B. ; Srikanth, G. ; Kumar, A. ; Saxena, R. ; Kapoor, V.K.

Dig Surg 2006;23:304-312

\section{3 胃贲门肿瘤的临床病理学特征及诊断}

Clinicopathological Characteristics and Prognosis of Carcinoma of the Gastric Cardia Kim, D.Y. ; Joo, J.K. ; Ryu, S.Y. ; $\quad$ Park, Y.K. ; Kim, Y.J. ; Kim, S.K.

Dig Surg 2006;23:313-318

319 CA199水平的上升对于未合并硬化性胆管炎的胆管癌患者是否有诊断意义?

Is a Raised CA 19-9 Level Diagnostic for a Cholangiocarcinoma in Patients with No History of Sclerosing Cholangitis ?

John, A.R. ; Haghighi, K.S. ; Taniere, P. ; Esmat, M.E. ; Tan, Y.M. ; Bramhall, S.R. Dig Surg 2006;23:319-324

325 近端胃切除术与全胃切除术分别相关的术后食管胆汁反流的差异

What Is the Difference between Proximal and Total Gastrectomy Regarding Postoperative Bile Reflux into the Oesophagus?

Katsoulis, I.E. ; Robotis, J.F. ; Kouraklis, G. ; Yannopoulos, P.A.

Dig Surg 2006;23:325-330

331 胸腺嘧啶磷酸化酶表达作为侵袭前壁的T3型直肠癌预后因子的重要性 Importance of Thymidine Phosphorylase Expression at the Invasive Front of T3 Rectal Cancer as a Prognostic Factor

Yoshimoto, K. ; Kawahara, H. ; Kobayashi, S. ; Kashiwagi, H. ; Hirai, K. ; Yanaga, K. Dig Surg 2006;23:331-335

336 重型急性胰腺炎患者肠内与胃肠外营养的随机化对照研究表明前者可明显降低死亡率 和胰腺感染性并发症

A Randomized Controlled Trial of Enteral versus Parenteral Feeding in Patients with Predicted Severe Acute Pancreatitis Shows a Significant Reduction in Mortality and in Infected Pancreatic Complications with Total Enteral Nutrition

Petrov, M.S. ; Kukosh, M.V. ; Emelyanov, N.V.

Dig Surg 2006;23:336-345

\section{综述 Review}

346 克罗恩氏病腹腔镜回结肠切除术效果的短期观察

Short-Term Outcomes after Laparoscopic lleocolic Resection for Crohn's Disease

Polle, S.W. ; Wind, J. ; Ubbink, D.T. ; Hommes, D.W. ; Gouma, D.J. ; Bemelman, W.A Dig Surg 2006;23:346-357

358 目前门腔分流术在肝静脉流出道阻塞处理中的地位

Current Role of Portosystemic Shunt Surgery in the Management of Hepatic Venous 


\section{Outflow Obstruction}

Singhal, D. ; de Castro, S. ; Goyal, N. ; Gouma, D.J. ; Chaudhary, A. ; van Gulik, T.M. Dig Surg 2006;23:358-369

370 腹腔镜胆囊切除术中的动脉损伤: 一个被低估了的难题 Vascular Injuries in Laparoscopic Cholecystectomy: An Underestimated Problem Tzovaras, G. ; Dervenis, C.

Dig Surg 2006;23:370-374

375 外科术前肠道准备：外科标准或已过时?

Preoperative Bowel Preparation: Surgical Standard or Past? Müller-Stich, B.P. ; Choudhry, A. ; Vetter, G. ; Antolovic, D. ; Mehrabi, A. ; Köninger, J. ; Weitz, J. ; Büchler, M.W.

Dig Surg 2006;23:375-380

\section{经验交流 How I Do It}

381 全部和近侧胃切除术后空肠袋重建预防反流

Antireflux Contrivance in Jejunal Pouch Reconstruction after Total and Proximal Gastrectomies

Ichikura, T. ; Chochi, K. ; Sugasawa, H. ; Mochizuki, H.

Dig Surg 2006;23:381-386 